\title{
Splitting methods for non-autonomous separable dynamical systems
}

\author{
S Blanes ${ }^{1}$ and F Casas $^{2}$ \\ ${ }^{1}$ Instituto de Matemática Multidisciplinar, Universitat Politécnica de Valencia, 46022-Valencia, \\ Spain \\ 2 Departament de Matemàtiques, Universitat Jaume I, 12071-Castellón, Spain \\ E-mail: serblaza@imm.upv.es and Fernando.Casas@uji.es
}

Received 1 September 2005, in final form 18 November 2005

Published 24 April 2006

Online at stacks.iop.org/JPhysA/39/5405

\begin{abstract}
A large number of splitting methods for autonomous separable systems exist in the literature which have been designed for many different structures of the vector field. However, the performance of most of these methods is diminished and their orders of accuracy are frequently reduced when applied to nonautonomous problems. Based on the formal solution obtained from the Magnus series expansion, we show how to modify a standard splitting method for autonomous problems to treat non-autonomous systems with similar or better efficiency. We illustrate this technique to build new fourth- and sixth-order schemes whose performance is then illustrated on several numerical examples.
\end{abstract}

PACS numbers: 02.60.-x, 02.60.Lj

Mathematics Subject Classification: 65L05, 65P10

(Some figures in this article are in colour only in the electronic version)

\section{Introduction}

Splitting methods constitute a widespread procedure to integrate numerically differential equations, particularly in the context of geometric integration. Suppose we have a system of ODEs of the form

$$
x^{\prime}=f(x)=f^{[A]}(x)+f^{[B]}(x), \quad x\left(t_{0}\right)=x_{0} \in \mathbb{R}^{d},
$$

with $f: \mathbb{R}^{d} \longrightarrow \mathbb{R}^{d}$ and such that the associated vector field (or Lie operator) is split accordingly as $L_{f}=L_{f^{[A]}}+L_{f^{[B]}}$, with

$$
L_{f^{[A]}}=\sum_{i=1}^{d} f_{i}^{[A]}(x) \frac{\partial}{\partial x_{i}}, \quad L_{f^{[B]}}=\sum_{i=1}^{d} f_{i}^{[B]}(x) \frac{\partial}{\partial x_{i}} .
$$


Suppose in addition that the $h$-flows $\varphi_{h}^{[A]}$ and $\varphi_{h}^{[B]}$ corresponding to $L_{f^{[A]}}$ and $L_{f^{[B]}}$, i.e., the solution of the systems

$$
x^{\prime}=f^{[A]}(x), \quad x^{\prime}=f^{[B]}(x),
$$

respectively, can be either exactly computed or accurately approximated. In that case it is useful to consider splitting methods of the form

$$
\psi_{h}=\varphi_{a_{1} h}^{[A]} \circ \varphi_{b_{1} h}^{[B]} \circ \varphi_{a_{2} h}^{[A]} \circ \cdots \circ \varphi_{b_{m-1} h}^{[B]} \circ \varphi_{a_{m} h}^{[A]} \circ \varphi_{b_{m} h}^{[B]}
$$

with $a_{i}, b_{i} \in \mathbb{R}$ chosen as to ensure that the numerical integrator $\psi_{h}$ is an approximation of order $p$ in the time step $h$ to the exact flow $\varphi_{h}$.

A large number of splitting methods of different orders exist in the literature (see $[10,13,17]$ and references therein) which are especially adapted to numerically integrate different families of problems. Thus, one could distinguish, for example (i) the general separable problem (1), when no more assumptions are made on the vector fields $L_{f^{[A]}}$ and $L_{f^{[B]}}$; (ii) near-integrable systems, which correspond to $x^{\prime}=f^{[A]}(x)+\varepsilon f^{[B]}(x)$ with $\|\varepsilon\| \ll 1$; (iii) the second-order differential equation $x^{\prime \prime}=g(x)$, etc. The performance of the different splitting schemes may greatly differ depending on the particular problem where they are used. It is important, then, to first analyse the problem to be solved in order to choose the most appropriate method.

If the problem is non-autonomous, i.e.,

$$
x^{\prime}=f(x, t)=f^{[A]}(x, t)+f^{[B]}(x, t), \quad x\left(t_{0}\right)=x_{0},
$$

the usual trick of taking $t$ as a new coordinate allows us to apply standard algorithms on the transformed (autonomous) equation. More specifically, equation (3) is equivalent to the enlarged system

$$
\frac{\mathrm{d}}{\mathrm{d} t}\left\{\begin{array}{c}
x \\
x_{t 1} \\
x_{t 2}
\end{array}\right\}=\underbrace{\left\{\begin{array}{c}
f^{[A]}\left(x, x_{t 1}\right) \\
0 \\
1
\end{array}\right\}}_{\hat{f}^{[A]}}+\underbrace{\left\{\begin{array}{c}
f^{[B]}\left(x, x_{t 2}\right) \\
1 \\
0
\end{array}\right\}}_{\hat{f}^{[B]}}
$$

with $x_{t 1}, x_{t 2} \in \mathbb{R}$. Note that if the systems

$$
y^{\prime}=\hat{f}^{[A]}(y), \quad y^{\prime}=\hat{f}^{[B]}(y)
$$

with $y=\left(x, x_{t 1}, x_{t 2}\right)$ are solvable, then a splitting method similar to (2) can be used, since $x_{t 1}$ is constant when integrating the first equation and $x_{t 2}$ is constant when solving the second one. This, in fact, can be considered as a generalization of the procedure proposed in [21] for timedependent and separable Hamiltonian systems, and is of interest if the time-dependent part in $f^{[A]}$ and $f^{[B]}$ is cheap to compute. Otherwise the overall algorithm may be computationally costly, since these functions have to be evaluated $m$ times (the number of stages in (2)) per time step.

Another drawback of the procedure is the following. Suppose that, when the time is frozen, the function $f$ in (3) has a special structure which allows us to apply highly efficient integrators. If now $t$ is a variable, with (4) this time dependency is eliminated but the structure of the equation is generally modified and one has to resort to more general and less efficient integrators. Let us illustrate this issue with a simple example.

Example. Consider the time-dependent Hamiltonian

$$
H(q, p, t)=\mathrm{e}^{-\epsilon t} \frac{1}{2} p^{2}+\mathrm{e}^{\epsilon t}\left(\frac{1}{4} q^{4}-\frac{1}{2} q^{2}-\delta \cos (\omega t) q\right),
$$



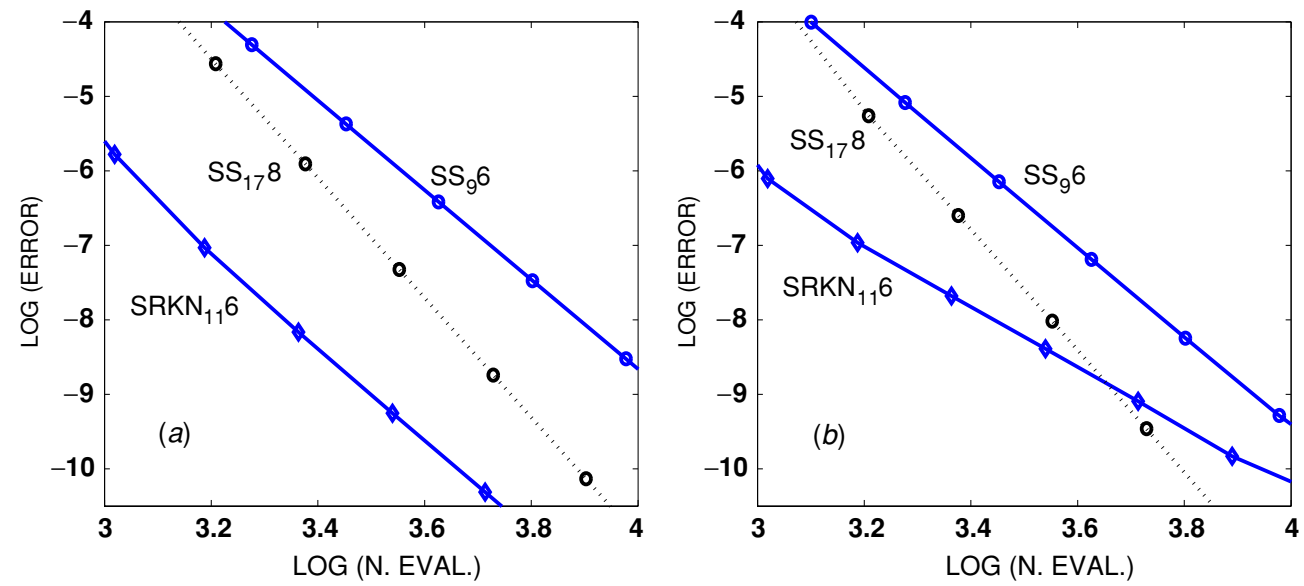

Figure 1. Average error versus number of force evaluations in the numerical integration of (6) using sixth- and eighth-order symplectic integrators for general separable systems $\left(\mathrm{SS}_{9} 6\right.$ and $\left.\mathrm{SS}_{17} 8\right)$ and a sixth-order symplectic integrator for Nyström problems $\mathrm{SRKN}_{11} 6$ with initial conditions $q(0)=1.75, p(0)=0 .(a) \epsilon=\delta=0 ;(b) \epsilon=1 / 20, \delta=1 / 4, \omega=1$.

$q, p \in \mathbb{R}$, with associated equations

$$
q^{\prime}=\mathrm{e}^{-\epsilon t} p, \quad p^{\prime}=-\mathrm{e}^{\epsilon t}\left(q^{3}-q-\delta \cos (\omega t)\right) .
$$

This system corresponds to the well-known Duffing oscillator [9]. Let us discuss first the autonomous case, $\epsilon=\delta=0$, and integrate the corresponding equations (6) with a symplectic method. In fact, since the kinetic energy is quadratic in momenta, symplectic Nyström methods usually show the best performance [20]. Thus, we carry out the integration in this case with the 9-stage sixth-order $\mathrm{SS}_{9} 6$ and the 17-stage eighth-order $\mathrm{SS}_{17} 8$ methods for general separable problems built by McLachlan [15], and the symmetric 11-stage sixth-order Nyström method $\mathrm{SRKN}_{11} 6$ given in [6].

We take as initial conditions $q(0)=1.75, p(0)=0$, integrate up to $t=10 \pi$ and measure the average error in phase space in terms of the number of force evaluations for different time steps (in logarithmic scale). The results are shown in figure 1(a). Observe how the especially adapted $\mathrm{SRKN}_{11} 6$ scheme is the most efficient method.

Next we repeat the same experiment with $\epsilon=1 / 20, \delta=1 / 4, \omega=1$ and the splitting (4). The corresponding results are plotted in figure $1(b)$. It is worth noting how the performance of the Nyström method has deteriorated. In fact, it now behaves just as a fourth-order scheme. In addition, each step involves 11 evaluations of the time-dependent functions.

To avoid the difficulties exhibited by the previous example, several numerical methods based on the Magnus series have been proposed recently [5, 7]. The idea is first to express the exact flow of $x^{\prime}=f(x, t)$ as the exponential of the Lie operator associated with a series of autonomous functions $w=\sum_{i} w_{i}$. Then this series is truncated, $w \approx w^{[p]}$, and at $t=t_{0}+h$ the $h$-flow of the autonomous differential equation

$$
x^{\prime}=w^{[p]}(x), \quad x(0)=x_{0}
$$

is approximated with a numerical integrator up to a certain order consistent with the previous truncation.

The purpose of this paper is to adapt the above procedure to the explicitly time-dependent separable system (3) and design new and efficient splitting methods for such a problem. More 
specifically, we propose to approximate the exact solution of (3) or equivalently the flow $\varphi_{h}$ by the composition

$$
\psi_{m, h}^{[p]}=\varphi_{h}^{\left[\hat{A}_{1}\right]} \circ \varphi_{h}^{\left[\hat{B}_{1}\right]} \circ \varphi_{h}^{\left[\hat{A}_{2}\right]} \circ \cdots \circ \varphi_{h}^{\left[\hat{B}_{m-1}\right]} \circ \varphi_{h}^{\left[\hat{A}_{m}\right]} \circ \varphi_{h}^{\left[\hat{B}_{m}\right]},
$$

where the maps $\varphi_{h}^{\left[\hat{A}_{i}\right]}, \varphi_{h}^{\left[\hat{B}_{i}\right]}$ are the exact $h$-flows corresponding to the time-independent differential equations

$$
x^{\prime}=\hat{A}_{i}(x), \quad x^{\prime}=\hat{B}_{i}(x), \quad i=1, \ldots, m
$$

respectively, with

$$
\hat{A}_{i}(x) \equiv \sum_{j=1}^{k} \rho_{i j} f^{[A]}\left(x, \tau_{j}\right), \quad \hat{B}_{i}(x) \equiv \sum_{j=1}^{k} \sigma_{i j} f^{[B]}\left(x, \tau_{j}\right) .
$$

Here $\tau_{j}=t_{0}+c_{j} h$ and the (real) constants $c_{j}, \rho_{i j}, \sigma_{i j}$ are chosen such that $\varphi_{h}=\psi_{m, h}^{[p]}+$ $\mathcal{O}\left(h^{p+1}\right)$. Furthermore, our aim is that the new schemes, when applied to (3) with the time frozen, reproduce the standard splitting (2). This will be accomplished by ensuring that $\sum_{j} \rho_{i j}=a_{i}$ and $\sum_{j} \sigma_{i j}=b_{i}$. The integrators of order 4 and 6 that we construct here satisfy this property and are generally more efficient than standard splitting methods applied to the enlarged system (4).

As we will show, the only restriction for $c_{j}$ in (10) is that they must be nodes of a quadrature rule of order at least $p$, and thus Newton-Cotes, Gauss-Legendre, Lobatto and Radau formulae are equally valid options. For instance, if a Gauss-Legendre quadrature rule is adopted, with $k$ evaluations of $f^{[A]}\left(x, \tau_{j}\right)$ and $f^{[B]}\left(x, \tau_{j}\right)$ a method of order $p=2 k$ can be built (taking $m$ sufficiently large). Once the quadrature nodes $\tau_{j}$ and the number of stages $m$ are fixed, there still remains to obtain the coefficients $\rho_{i j}, \sigma_{i j}$ such that $\psi_{m, h}^{[p]}$ has the desired order. This is done by requiring that composition (8) match the solution of (3) as given by the Magnus expansion. The task is made easier by noting that the order conditions to be satisfied by $\rho_{i j}$ and $\sigma_{i j}$ are identical both for linear and nonlinear vector fields. Thus, we first solve the problem for the linear case and then we generalize the treatment to arbitrary nonlinear separable problems.

The basic assumption in this approach is, obviously, that equations (9) are either solvable or accurately and efficiently approximated. Relevant examples of this situation include: (i) classical Hamiltonian systems with a time-dependent potential, (ii) the linear and nonlinear Schrödinger equations with time-dependent kinetic and/or potential energy and (iii) the linear system $x^{\prime}=M(t) x$ when the matrix $M$ is split into its upper and lower triangular parts.

The class of methods we propose here is closely related to the schemes analysed in [7] for the general equation $x^{\prime}=f(x, t)$. There the exact flow $\varphi_{h}$ is approximated by the composition

$$
\psi_{h}^{\left[f_{1}\right]} \circ \psi_{h}^{\left[f_{2}\right]} \circ \cdots \circ \psi_{h}^{\left[f_{n-1}\right]} \circ \psi_{h}^{\left[f_{n}\right]},
$$

where $\psi_{h}^{\left[f_{i}\right]}$ is a map which approximates up to order $p$ the solution of the system

$$
x^{\prime}=f_{i}(x) \equiv \sum_{j=1}^{k} d_{i j} f\left(x, \tau_{j}\right), \quad i=1, \ldots, n
$$

for some constants $\tau_{j}, d_{i j}$.

If the scheme (11) is applied to the separable problem (3), then each map $\psi_{h}^{\left[f_{i}\right]}$ may be chosen as a splitting method, since (12) is also separable. Observe, though, that this splitting method has to be used $n$ times per step and this is exceedingly costly for a number of problems. 


\section{Magnus expansion for separable linear systems}

As stated in the introduction, we first consider the simpler case of a linear time-dependent separable problem

$$
x^{\prime}=M(t) x=(A(t)+B(t)) x, \quad x\left(t_{0}\right)=x_{0}
$$

instead of (3) and design the new algorithms for (13). Here $A(t)$ and $B(t)$ are sufficiently smooth $d \times d$ matrices, so that the maps appearing in (8) (or (2)) are simply exponentials of linear combinations of $A$ and $B$ evaluated at $t=\tau_{j}, j=1, \ldots, k$.

It has been known for a long time that, locally, the solution of (13) can be written as

$$
x\left(t_{0}+h\right)=\mathrm{e}^{\Omega(h)} x_{0},
$$

where $\Omega(h)=\sum_{k=1}^{\infty} \Omega_{k}(h)$ and $\Omega_{k}$ is a multiple integral of combinations of nested commutators containing $k$ matrices $M(t)$ [14]. This is the so-called Magnus expansion of the solution. An important feature of this expansion is that if (13) is defined on a Lie group $\mathcal{G}, \mathrm{e}^{\Omega}$ stays on $\mathcal{G}$ even if the series is truncated, provided $M(t)$ belongs to the Lie algebra associated with $\mathcal{G}$ [11]. In [12] it is shown how all the multidimensional integrals appearing in $\Omega$ can be approximated just by evaluating $M(t)$ at the nodes of a one-dimensional quadrature. Also the number of commutators has been reduced to a minimum, thus producing powerful numerical integrators [2, 3].

If $M(t)$ is separable, as in (13), the presence of commutators in methods of order higher than 2 typically destroys this separability and, as a consequence, the evaluation of the exponential is more complicated [4]. This is especially serious for nonlinear problems, since then the commutators are replaced by Lie brackets of vector fields. The new schemes we propose here also avoid this deficiency.

When a Taylor series of the matrix $M(t)$ is inserted in the recurrence defining the Magnus expansion, one gets explicitly the expression of $\Omega_{k}$. To take advantage of the time-symmetry property of the solution, the Taylor series is considered around the midpoint $t_{1 / 2}=t_{0}+h / 2$. In that case only odd powers of $h$ appear in $\Omega$. Specifically, up to order $p \equiv 2 s=6$ the relevant terms in $\Omega$ are [2]

$$
\begin{array}{r}
\Omega=\mu_{1}+\frac{1}{12} \mu_{3}-\frac{1}{12}\left[\mu_{1}, \mu_{2}\right]+\frac{1}{240}\left[\mu_{2}, \mu_{3}\right]+\frac{1}{360}\left[\mu_{1}, \mu_{1}, \mu_{3}\right] \\
-\frac{1}{240}\left[\mu_{2}, \mu_{1}, \mu_{2}\right]+\frac{1}{720}\left[\mu_{1}, \mu_{1}, \mu_{1}, \mu_{2}\right]+\mathcal{O}\left(h^{7}\right),
\end{array}
$$

where $\mu_{i}=\left.\frac{h^{i}}{(i-1) !} \frac{d^{i-1} M(t)}{\mathrm{d} t^{i-1}}\right|_{t=t_{1 / 2}}$ and $\left[\mu_{i_{1}}, \ldots, \mu_{i_{l-1}}, \mu_{i_{l}}\right] \equiv\left[\mu_{i_{1}},\left[\ldots,\left[\mu_{i_{l-1}}, \mu_{i_{l}}\right] \ldots\right]\right]$ is an element of order $\mathcal{O}\left(h^{i_{1}+\cdots+i_{l}}\right)$. As is well known, $\mu_{1}, \mu_{2}, \mu_{3}$ can be considered as the generators (with grades $1,2,3$, respectively) of a graded free Lie algebra $\mathcal{L}\left(\mu_{1}, \mu_{2}, \mu_{3}\right)$ [18].

Let us now introduce the averaged matrices

$$
M^{(i)} \equiv \frac{1}{h^{i}} \int_{t_{0}}^{t_{0}+h}\left(t-t_{1 / 2}\right)^{i} M(t) \mathrm{d} t=\frac{1}{h^{i}} \int_{-h / 2}^{h / 2} t^{i} M\left(t+t_{1 / 2}\right) \mathrm{d} t
$$

for $i=0, \ldots, s-1$. If their analytical evaluation is not possible or is computationally expensive, a numerical quadrature may be used instead. In fact, the integrals $M^{(i)}, i \geqslant 1$, can be approximated up to the required order just by evaluating $M$ at the nodes $c_{i}$ of the quadrature rule required to compute $M^{(0)}$. Denoting these by $M_{i} \equiv M\left(t_{0}+c_{i} h\right), i=1, \ldots, k$, one can write

$$
M^{(i)}=h \sum_{j=1}^{k}\left(Q_{X}^{(s, k)}\right)_{i j} M_{j}, \quad i=0, \ldots, s-1
$$


with

$$
Q_{X}^{(s, k)}=\left(\begin{array}{ccc}
b_{1} & \cdots & b_{k} \\
\vdots & \ddots & \vdots \\
b_{1}\left(c_{1}-\frac{1}{2}\right)^{s-1} & \cdots & b_{k}\left(c_{k}-\frac{1}{2}\right)^{s-1}
\end{array}\right)
$$

and $\left(Q_{X}^{(s, k)}\right)_{i j}$ stands for the element $i j$ of the matrix $Q_{X}^{(s, k)} \in \mathbb{R}^{s \times k}$.

Here $b_{i}, c_{i}$ are the weights and nodes, respectively, of the quadrature $X$. It should be remarked that in (17) we have omitted terms of order greater than the order of the method itself.

As an example, if fourth- and sixth-order Gauss-Legendre quadrature rules are considered, then $b_{1}=b_{2}=1 / 2, c_{1}=1 / 2-\sqrt{3} / 6, c_{2}=1 / 2+\sqrt{3} / 6$ to order 4 and $b_{1}=b_{3}=5 / 18$, $b_{2}=4 / 9, c_{1}=1 / 2-\sqrt{15} / 10, c_{2}=1 / 2, c_{3}=1 / 2+\sqrt{15} / 10$ to order 6 , so that

$$
Q_{G}^{(2,2)}=\left(\begin{array}{cc}
\frac{1}{2} & \frac{1}{2} \\
-\frac{\sqrt{3}}{12} & \frac{\sqrt{3}}{12}
\end{array}\right), \quad Q_{G}^{(3,3)}=\left(\begin{array}{ccc}
\frac{5}{18} & \frac{4}{9} & \frac{5}{18} \\
-\frac{\sqrt{15}}{36} & 0 & \frac{\sqrt{15}}{36} \\
\frac{1}{24} & 0 & \frac{1}{24}
\end{array}\right) .
$$

On the other hand, it is clear that $M^{(i)}(-h)=(-1)^{i+1} M^{(i)}(h)$ and furthermore

$$
M^{(i)}=\sum_{j=1}^{s}\left(T^{(s)}\right)_{i j} \mu_{j} \equiv \sum_{j=1}^{s} \frac{1-(-1)^{i+j}}{(i+j) 2^{i+j}} \mu_{j}, \quad 0 \leqslant i \leqslant s-1 .
$$

If this relation is inverted (to order $4, s=2$, and six, $s=3$ ) one has

$$
R^{(2)} \equiv\left(T^{(2)}\right)^{-1}=\left(\begin{array}{cc}
1 & 0 \\
0 & 12
\end{array}\right), \quad R^{(3)}=\left(\begin{array}{ccc}
\frac{9}{4} & 0 & 15 \\
0 & 12 & 0 \\
-15 & 0 & 180
\end{array}\right)
$$

and the corresponding expression of $\mu_{j}$ in terms of $M^{(i)}$ or $M_{j}$ is then given by

$$
\mu_{i}=\sum_{j=1}^{s}\left(R^{(s)}\right)_{i j} M^{(j-1)}=h \sum_{j=1}^{k}\left(R^{(s)} Q_{X}^{(s, k)}\right)_{i j} M_{j}
$$

In other words, by virtue of (22) we can write $\Omega(h)$ in terms of the univariate integrals (16) and also in terms of any desired quadrature rule, although the process of construction and analysis of the new methods is considerably simplified if one works in the free Lie algebra $\mathcal{L}\left(\mu_{1}, \mu_{2}, \mu_{3}\right)$.

Let us discuss specifically the separable problem (13). It is clear that $\mu_{i}=\alpha_{i}+\beta_{i}$, where

$$
\alpha_{i}=\left.\frac{h^{i}}{(i-1) !} \frac{\mathrm{d}^{i-1} A(t)}{\mathrm{d} t^{i-1}}\right|_{t=t_{1 / 2}}, \quad \beta_{i}=\left.\frac{h^{i}}{(i-1) !} \frac{\mathrm{d}^{i-1} B(t)}{\mathrm{d} t^{i-1}}\right|_{t=t_{1 / 2}}
$$

and thus, from (15),

$\Omega(h)=\alpha_{1}+\beta_{1}+\frac{1}{12}\left(\alpha_{3}+\beta_{3}\right)+\frac{1}{12}\left(-\left[\alpha_{1}, \alpha_{2}\right]-\left[\alpha_{1}, \beta_{2}\right]+\left[\alpha_{2}, \beta_{1}\right]-\left[\beta_{1}, \beta_{2}\right]\right)+\mathcal{O}\left(h^{5}\right)$,

whereas at higher orders the number of terms increases dramatically. It makes sense, then, to impose some simplifying conditions. For instance, if we suppose that the matrices $A(t)$ and $B(t)$ commute with themselves at different times, then obviously $\left[\alpha_{i}, \alpha_{j}\right]=\left[\beta_{i}, \beta_{j}\right]=0$, 
Table 1. Dimension of the subspace $\mathcal{L}_{p}$ of the graded Lie algebra generated by $\left\{\alpha_{1}, \beta_{1}, \alpha_{2}, \beta_{2}, \alpha_{3}, \beta_{3}\right\}$ when: (i) the algebra is free $\left(c_{p, 1}\right)$, (ii) $\left[\alpha_{i}, \alpha_{j}\right]=\left[\beta_{i}, \beta_{j}\right]=0$ for $i \neq j$ $\left(c_{p, 2}\right)$, (iii) in addition $\left[\beta_{i}, \beta_{j}, \beta_{k}, \alpha_{l}\right]=0\left(c_{p, 3}\right)$ and (iv) finally $\alpha_{i}=0$ for $i>1\left(c_{p, 4}\right)$.

\begin{tabular}{rrrrr}
\hline$p$ & $c_{p, 1}$ & $c_{p, 2}$ & $c_{p, 3}$ & $c_{p, 4}$ \\
\hline 1 & 2 & 2 & 2 & 2 \\
2 & 3 & 3 & 3 & 2 \\
3 & 8 & 6 & 6 & 4 \\
4 & 16 & 10 & 9 & 6 \\
5 & 42 & 24 & 20 & 13 \\
\hline
\end{tabular}

$i \neq j$. In that case (15) leads to

$$
\begin{aligned}
\Omega(h)=\alpha_{1}+\beta_{1} & +\frac{1}{12}\left(\alpha_{3}+\beta_{3}\right)+\frac{1}{12}\left(-\left[\alpha_{1}, \beta_{2}\right]+\left[\alpha_{2}, \beta_{1}\right]\right)+\frac{1}{240}\left(\left[\alpha_{2}, \beta_{3}\right]-\left[\alpha_{3}, \beta_{2}\right]\right) \\
& +\frac{1}{360}\left(\left[\alpha_{1}, \alpha_{1}, \beta_{3}\right]-\left[\alpha_{1}, \alpha_{3}, \beta_{1}\right]+\left[\beta_{1}, \alpha_{1}, \beta_{3}\right]-\left[\beta_{1}, \alpha_{3}, \beta_{1}\right]\right) \\
& +\frac{1}{240}\left(\left[\alpha_{1}, \alpha_{2}, \beta_{2}\right]+\left[\alpha_{2}, \alpha_{2}, \beta_{1}\right]-\left[\beta_{2}, \alpha_{1}, \beta_{2}\right]+\left[\beta_{1}, \alpha_{2}, \beta_{2}\right]\right) \\
& +\frac{1}{720}\left(\left[\alpha_{1}, \alpha_{1}, \alpha_{1}, \beta_{2}\right]-\left[\alpha_{1}, \alpha_{1}, \alpha_{2}, \beta_{1}\right]+\left[\alpha_{1}, \beta_{1}, \alpha_{1}, \beta_{2}\right]\right. \\
& -\left[\alpha_{1}, \beta_{1}, \alpha_{2}, \beta_{1}\right]+\left[\beta_{1}, \alpha_{1}, \alpha_{1}, \beta_{2}\right]-\left[\beta_{1}, \alpha_{1}, \alpha_{2}, \beta_{1}\right] \\
& \left.+\left[\beta_{1}, \beta_{1}, \alpha_{1}, \beta_{2}\right]-\left[\beta_{1}, \beta_{1}, \alpha_{2}, \beta_{1}\right]\right)+\mathcal{O}\left(h^{7}\right) .
\end{aligned}
$$

For Nyström problems, in addition, $\left[\beta_{i}, \beta_{j}, \beta_{k}, \alpha_{l}\right]=0$. This reduces further the number of terms in $\Omega(h)$. Finally, the special case $x^{\prime}=(A+B(t)) x$ is also worth to be studied separately. Then $\alpha_{i}=0$ for $i>1$ in the expression (24). For illustration, in table 1 we collect the dimensions of the first homogeneous subspaces $\mathcal{L}_{p}$ of the graded Lie algebra generated by $\left\{\alpha_{1}, \beta_{1}, \alpha_{2}, \beta_{2}, \alpha_{3}, \beta_{3}\right\}$ for each case. The column $c_{p, 1}$ indicates the dimension in the general case, computed according to the (generalized) Witt formula [18]. It exhibits clearly the necessity of imposing restrictions to reduce the complexity of the problem.

Of course, expressions (23) and (24) can also be written in terms of the univariate integrals

$$
A^{(i)} \equiv \frac{1}{h^{i}} \int_{-h / 2}^{h / 2} t^{i} A\left(t+t_{1 / 2}\right) \mathrm{d} t, \quad B^{(i)} \equiv \frac{1}{h^{i}} \int_{-h / 2}^{h / 2} t^{i} B\left(t+t_{1 / 2}\right) \mathrm{d} t,
$$

through linear relations analogous to (22).

\section{Splitting methods from the Magnus expansion}

As it was stated in the introduction, the idea now is to reproduce $\mathrm{e}^{\Omega(h)}$ with a composition of the form

$$
\psi_{m, h}^{[p]}=\prod_{i=1}^{m} \mathrm{e}^{\tilde{A}_{i}} \mathrm{e}^{\tilde{B}_{i}}
$$

with

$$
\tilde{A}_{i}=a_{i 1} \alpha_{1}+a_{i 2} \alpha_{2}, \quad \tilde{B}_{i}=b_{i 1} \beta_{1}+b_{i 2} \beta_{2} \quad \text { for } \quad p \equiv 2 s=4
$$

and

$\tilde{A}_{i}=a_{i 1} \alpha_{1}+a_{i 2} \alpha_{2}+a_{i 3} \alpha_{3}, \quad \tilde{B}_{i}=b_{i 1} \beta_{1}+b_{i 2} \beta_{2}+b_{i 3} \beta_{3} \quad$ for $\quad p=6$. 
Introducing the matrices $A^{(m, s)}, B^{(m, s)} \in \mathbb{R}^{m \times s}$ with elements $\left(A^{(m, s)}\right)_{i j} \equiv a_{i j}$, $\left(B^{(m, s)}\right)_{i j} \equiv b_{i j}$, respectively, it is clear that

$$
\tilde{A}_{i}=\sum_{j=1}^{s}\left(A^{(m, s)}\right)_{i j} \alpha_{j}, \quad \tilde{B}_{i}=\sum_{j=1}^{s}\left(B^{(m, s)}\right)_{i j} \beta_{j}, \quad 1 \leqslant i \leqslant m .
$$

Now, taking into account relations (22), we have

$$
\tilde{A}_{i}=h \sum_{j=1}^{k}\left(R^{(m, k)}\right)_{i j} A_{j}, \quad \tilde{B}_{i}=h \sum_{j=1}^{k}\left(S^{(m, k)}\right)_{i j} B_{j}, \quad 1 \leqslant i \leqslant m
$$

with

$$
R^{(m, k)} \equiv A^{(m, s)} R^{(s)} Q_{X}^{(s, k)}, \quad S^{(m, k)} \equiv B^{(m, s)} R^{(s)} Q_{X}^{(s, k)},
$$

matrices $m \times k$ with elements $\left(R^{(m, k)}\right)_{i j} \equiv \rho_{i j},\left(S^{(m, k)}\right)_{i j} \equiv \sigma_{i j}$, respectively. In (30), $A_{j} \equiv A\left(t_{0}+c_{j} h\right)$ and $B_{j} \equiv B\left(t_{0}+c_{j} h\right)$, the matrices $A$ and $B$ evaluated at the nodes of the chosen quadrature rule $X$.

Of course the coefficients $a_{i j}, b_{i j}$ are chosen as to guarantee that $\psi_{m, h}^{[p]} x_{0}$ renders an approximation of order $p$ to the exact solution. The usual approach consists in expanding (26) with the Baker-Campbell-Hausdorff $(\mathrm{BCH})$ formula [23], matching with the corresponding expression of $\Omega(h)$ (e.g. (23) or (24)) to determine the order conditions and solving these equations to find the coefficients of the composition.

The number of order conditions for a method of order $p$ is precisely $\operatorname{dim} \mathcal{L}_{1}+\cdots+\operatorname{dim} \mathcal{L}_{p}$ and, as shown in table 1 , this number increases rapidly with $p$. In consequence, it seems convenient to adopt some simplifying assumptions.

Note that the scheme (26) is a natural extension to the non-autonomous system (13) of the standard splitting method

$$
\psi_{h}=\prod_{i=1}^{m} \mathrm{e}^{h a_{i} A} \mathrm{e}^{h b_{i} B},
$$

for the time-independent problem $x^{\prime}=(A+B) x$. In fact, if $\psi_{m, h}^{[p]}$ is applied to this equation, then $\alpha_{2}=\beta_{2}=\alpha_{3}=\beta_{3}=0$, since there is no time dependence and $\tilde{A}_{i}=h a_{i 1} A, \tilde{B}_{i}=h b_{i 1} B$. Therefore, one recovers the splitting method $\psi_{h}$ by taking $a_{i}=a_{i 1}, b_{i}=b_{i 1}, i=1, \ldots, m$ in (26).

The above observation justifies our first simplifying assumption. We start from a good splitting method for the system (13) when the $t$ variable is frozen. This sets up the values of $a_{i 1}, b_{i 1}$ and so only the order conditions involving $a_{i j}, b_{i j}$ with $j \geqslant 2$ have to be analysed. An added value of this hypothesis is that the most difficult order conditions, which are precisely those involving only $a_{i 1}, b_{i 1}$, are already solved by the specific composition (32) considered at the beginning.

The second simplifying assumption consists in imposing the time symmetry of $\Omega(h)$ to the composition (26). This symmetry is automatically satisfied (and thus all order conditions at even orders) if the coefficients are such that either

$$
a_{m+1-i, j}=(-1)^{j+1} a_{i j}, \quad b_{m-i, j}=(-1)^{j+1} b_{i j}, \quad b_{m j}=0,
$$

or

$$
a_{1 j}=0, \quad a_{m+1-i, j}=(-1)^{j+1} a_{i+1, j}, \quad b_{m+1-i, j}=(-1)^{j+1} b_{i j}
$$

for $j=1,2,3, i=1,2, \ldots, m$, depending on whether the composition (26) starts with $\mathrm{e}^{\tilde{A}_{1}}$ or $\mathrm{e}^{\tilde{B}_{1}}$, respectively. In the first case, the scheme will be said to be of type ABA, whereas in the 
second, of type BAB. Since the first or last exponential is cancelled and one exponential can be concatenated in two consecutive steps (first same as last, FSAL, property) these symmetric schemes are referred to as $(m-1)$-stage methods.

With the previous premises fourth-order methods can be obtained very easily and even for sixth-order schemes the number of order conditions to be solved is not prohibitively large.

\subsection{Fourth-order methods}

For simplicity we consider first the case $\left[\alpha_{1}, \alpha_{2}\right]=\left[\beta_{1}, \beta_{2}\right]=0$ in (23) and take the composition

$$
\psi_{m, h}^{[4]}=\prod_{i=1}^{m} \mathrm{e}^{\tilde{A}_{i}} \mathrm{e}^{\tilde{B}_{i}}=\prod_{i=1}^{m} \mathrm{e}^{a_{i 1} \alpha_{1}+a_{i 2} \alpha_{2}} \mathrm{e}^{b_{i 1} \beta_{1}+b_{i 2} \beta_{2}},
$$

$a_{i 1}, b_{i 1}$ being the parameters of a standard splitting method of order 4 for autonomous problems. Therefore, the consistency conditions

$$
\sum_{i=1}^{m} a_{i 1}=1, \quad \sum_{i=1}^{m} b_{i 1}=1
$$

hold. When the $\mathrm{BCH}$ formula is applied to (35) one gets $\mathrm{e}^{C(\lambda)}$, where

$$
\begin{aligned}
C(\boldsymbol{\lambda})=\lambda_{1} \alpha_{1}+ & \lambda_{2} \beta_{1}+\lambda_{3} \alpha_{2}+\lambda_{4} \beta_{2}+\lambda_{5}\left[\alpha_{1}, \beta_{1}\right]+\lambda_{6}\left[\alpha_{1}, \beta_{2}\right] \\
& +\lambda_{7}\left[\alpha_{2}, \beta_{1}\right]+\lambda_{8}\left[\alpha_{1}, \alpha_{1}, \beta_{1}\right]+\lambda_{9}\left[\beta_{1}, \alpha_{1}, \beta_{1}\right]+\lambda_{10}\left[\beta_{1}, \alpha_{2}, \beta_{1}\right] \\
& +\lambda_{11}\left[\alpha_{2}, \beta_{2}\right]+\lambda_{12}\left[\alpha_{1}, \alpha_{1}, \beta_{2}\right]+\lambda_{13}\left[\alpha_{1}, \alpha_{2}, \beta_{1}\right]+\lambda_{14}\left[\beta_{1}, \alpha_{1}, \beta_{2}\right] \\
& +\lambda_{15}\left[\alpha_{1}, \alpha_{1}, \alpha_{1}, \beta_{1}\right]+\lambda_{16}\left[\alpha_{1}, \beta_{1}, \alpha_{1}, \beta_{1}\right]+\lambda_{17}\left[\beta_{1}, \beta_{1}, \alpha_{1}, \beta_{1}\right] .
\end{aligned}
$$

Time symmetry forces that $\lambda_{3}, \lambda_{4}, \lambda_{5}, \lambda_{10}, \ldots, \lambda_{17}$ vanish, whereas the equations for $\lambda_{1}, \lambda_{2}, \lambda_{8}$ and $\lambda_{9}$ are satisfied by the starting fourth-order splitting method. In consequence, only the equations for $\lambda_{6}$ and $\lambda_{7}$ remain to be solved. If we denote

$$
s_{i}=\sum_{j=1}^{i} a_{j 1}, \quad u_{i}=\sum_{j=i}^{m} b_{j 1}
$$

$\left(s_{0}=0, s_{m}=1, u_{1}=1, u_{m+1}=0\right)$, then these equations are explicitly

$$
\sum_{i=1}^{m} b_{i 2} s_{i}=-\frac{1}{12}, \quad \sum_{i=1}^{m} a_{i 2} u_{i}=\frac{1}{12}
$$

where the symmetry conditions (33), (34) have still to be imposed. Perhaps the simplest solution is obtained by taking $a_{i 2}=b_{i 2}=0$ for $i>1$, i.e., with

$$
\mathrm{e}^{a_{11} \alpha_{1}+a_{12} \alpha_{2}} \mathrm{e}^{b_{11} \beta_{1}+b_{12} \beta_{2}} \mathrm{e}^{a_{21} \alpha_{1}} \mathrm{e}^{b_{21} \beta_{1}} \cdots \mathrm{e}^{b_{21} \beta_{1}} \mathrm{e}^{a_{21} \alpha_{1}} \mathrm{e}^{b_{11} \beta_{1}-b_{12} \beta_{2}} \mathrm{e}^{a_{11} \alpha_{1}-a_{12} \alpha_{2}}
$$

although this is not necessarily the most efficient distribution of the coefficients. Since $b_{m 1}=0$ in the previous composition, equations (36) lead to

$$
b_{12}\left(a_{11}-s_{m-1}\right)=b_{12}\left(2 a_{11}-1\right)=\frac{-1}{12} \quad \Longrightarrow \quad b_{12}=\frac{-1}{12\left(2 a_{11}-1\right)} ; \quad a_{12}=\frac{1}{12} \text {. }
$$

The first step in this procedure is to have an efficient standard splitting method. Observe that if $\alpha_{1}, \beta_{1}$ are the dominant terms and the main source of errors comes precisely from these terms, then one can use higher order standard splitting methods (of order 6 or 8) with the fourth-order Magnus approximation. The overall order of the resulting schemes will be still 4 , 
Table 2. Splitting methods of order 4 for separable non-autonomous systems. $\mathrm{GS}_{6}-4$ is intended for general separable problems, whereas $\mathrm{MN}_{6}-4$ is a Runge-Kutta-Nyström method. With the coefficients $a_{i j}, b_{i j}$ the matrices $A^{(m, 2)}, B^{(m, 2)}$, respectively, are formed.

\begin{tabular}{lll}
\hline \multicolumn{1}{c}{ Order $4 ; \mathrm{GS}_{6}-4$ Type BAB } \\
\hline$b_{11}=0.0792036964311957$ & $b_{12}=\left(2 a_{11}+2 a_{21}-2 b_{11}-b_{21}\right) / d$ \\
$a_{11}=0.209515106613362$ & $a_{12}=\left(2 a_{11}+2 a_{21}+a_{31}-2 b_{11}-2 b_{21}\right) / c$ \\
$b_{21}=0.353172906049774$ & $b_{22}=\left(-2 a_{11}+b_{11}\right) / d$ \\
$a_{21}=-0.143851773179818$ & $a_{22}=0$ \\
$b_{31}=-0.0420650803577195$ & $b_{32}=0$ \\
$a_{31}=1 / 2-\left(a_{11}+a_{21}\right)$ & $a_{32}=-a_{11} / c$ \\
$b_{41}=1-2\left(b_{11}+b_{21}+b_{31}\right)$ & $b_{42}=0$ \\
\hline \multicolumn{1}{c}{ Order $4 ; \mathrm{MN}_{6}-4$ Type BAB } \\
\hline$b_{11}=0.0829844064174052$ & $b_{12}=\left(2 a_{11}+2 a_{21}-2 b_{11}-b_{21}\right) / d$ \\
$a_{11}=0.245298957184271$ & $a_{12}=\left(2 a_{11}+2 a_{21}+a_{31}-2 b_{11}-2 b_{21}\right) / c$ \\
$b_{21}=0.396309801498368$ & $b_{22}=\left(-2 a_{11}+b_{11}\right) / d$ \\
$a_{21}=0.604872665711080$ & $a_{22}=0$ \\
$b_{31}=-0.0390563049223486$ & $b_{32}=0$ \\
$a_{31}=1 / 2-\left(a_{11}+a_{21}\right)$ & $a_{32}=-a_{11} / c$ \\
$b_{41}=1-2\left(b_{11}+b_{21}+b_{31}\right)$ & $b_{42}=0$ \\
$c=12\left(a_{11}+2 a_{21}+a_{31}-2 b_{11}+2 a_{11} b_{11}-2 b_{21}+2 a_{11} b_{21}\right)$ \\
$d=12\left(2 a_{21}-b_{11}+2 a_{11} b_{11}-2 a_{21} b_{11}-b_{21}+2 a_{11} b_{21}\right)$
\end{tabular}

but the error will presumably decrease. A situation where this approach is worth to be considered is when the time-dependent functions evolve smoothly.

In the general case of (23) we have two additional order conditions for the coefficients $a_{i 2}, b_{i 2}$ :

$$
\sum_{i=1}^{m} a_{i 2}\left(s_{i}+s_{i-1}\right)=-\frac{1}{6}, \quad \sum_{i=1}^{m} b_{i 2}\left(u_{i}+u_{i+1}\right)=\frac{1}{6} .
$$

These equations, together with (36), have to be solved for achieving a fourth-order integrator. When the symmetry (33), (34) is incorporated, the composition (35) requires at least five stages. In practice, efficient standard splitting methods involve a larger number of stages.

In table 2 we collect the coefficients for the splitting method $\mathrm{GS}_{6}-4$ for general separable problems, formed from the 6-stage $(m=7)$ fourth-order symmetric integrator $S_{6}$ of [6] (the role of $A$ and $B$ is interchanged for convenience in the presentation). When $\left[\beta_{i}, \beta_{j}, \beta_{k}, \alpha_{l}\right]=0$ we have the scheme $\mathrm{MN}_{6}-4$, built from the Runge-Kutta-Nyström (RKN) method $\mathrm{SRKN}_{6}^{b}$ of [6]. Both time-symmetric integrators have the BAB structure

$$
\mathrm{e}^{b_{11} \beta_{1}+b_{12} \beta_{2}} \mathrm{e}^{a_{11} \alpha_{1}+a_{12} \alpha_{2}} \mathrm{e}^{b_{21} \beta_{1}+b_{22} \beta_{2}} \cdots \mathrm{e}^{b_{21} \beta_{1}-b_{22} \beta_{2}} \mathrm{e}^{a_{11} \alpha_{1}-a_{12} \alpha_{2}} \mathrm{e}^{b_{11} \beta_{1}-b_{12} \beta_{2}}
$$

and the solutions collected in table 2 minimize the value of $\sum_{i}\left|a_{i 2}\right|$ and $\sum_{i}\left|b_{i 2}\right|$.

Particularizing to this case the linear relations (30) and (31) with the fourth-order Gaussian quadrature $G$, one has

$$
\tilde{A}_{i}=h \sum_{j=1}^{2}\left(A^{(m, 2)} R^{(2)} Q_{G}^{(2,2)}\right)_{i j} A_{j}, \quad \tilde{B}_{i}=h \sum_{j=1}^{2}\left(B^{(m, 2)} R^{(2)} Q_{G}^{(2,2)}\right)_{i j} B_{j},
$$


Table 3. Splitting methods of order 6 for separable non-autonomous systems. $\mathrm{GS}_{10}-6$ is intended for general separable problems, whereas $\mathrm{MN}_{11}-6$ is a Runge-Kutta-Nyström method. With the coefficients $a_{i j}, b_{i j}$ the matrices $A^{(m, 3)}, B^{(m, 3)}$ can be formed.

\begin{tabular}{|c|c|c|}
\hline \multicolumn{3}{|c|}{ Order $6 ; \mathrm{GS}_{10}-4$ Type ABA } \\
\hline$a_{11}=0.0502627644003922$ & $a_{12}=0.022059009674017884$ & $a_{13}=-0.000326878764898432$ \\
\hline$b_{11}=0.148816447901042$ & $b_{12}=0.06325193140810957$ & $b_{13}=0.03156029484304291$ \\
\hline$a_{21}=0.413514300428344$ & $a_{22}=0.03639087263834154$ & $a_{23}=0.05639771119273678$ \\
\hline$b_{21}=-0.132385865767784$ & $b_{22}=-0.0564220584435047$ & $b_{23}=0.00004713758165544868$ \\
\hline$a_{31}=0.0450798897943977$ & $a_{32}=-0.029722051174027396$ & $a_{33}=0.0032603041391350658$ \\
\hline$b_{31}=0.067307604692185$ & $b_{32}=0.030997085102486225$ & $b_{33}=0.001271609241968303$ \\
\hline$a_{41}=-0.188054853819569$ & $a_{42}=0.07316095552711696$ & $a_{43}=-0.008$ \\
\hline$b_{41}=0.432666402578175$ & $b_{42}=0.086709890573243$ & $b_{43}=0.012967625$ \\
\hline$a_{51}=0.541960678450780$ & $a_{52}=-0.10825317547305482$ & $a_{53}=0$ \\
\hline$b_{51}=1 / 2-\left(b_{11}+\cdots+b_{41}\right)$ & $b_{52}=0$ & $b_{53}=-0.00418$ \\
\hline$a_{61}=1-2\left(a_{11}+\cdots+a_{51}\right)$ & $a_{62}=0$ & $a_{63}=-0.019328939800613495$ \\
\hline \multicolumn{3}{|c|}{ Order $6 ; \mathrm{MN}_{11}-6$ Type BAB } \\
\hline$b_{11}=0.0414649985182624$ & $b_{12}=0.020732500126731092$ & $b_{13}=0.013608659602613978$ \\
\hline$a_{11}=0.123229775946271$ & $a_{12}=0.05402209364412427$ & $a_{13}=0.026164309515298998$ \\
\hline$b_{21}=0.198128671918067$ & $b_{22}=0.07464898167304031$ & $b_{23}=0.017248381524744054$ \\
\hline$a_{21}=0.290553797799558$ & $a_{22}=0.06726123187463062$ & $a_{23}=0.025979253037549735$ \\
\hline$b_{31}=-0.0400061921041533$ & $b_{32}=-0.0034491907957761338$ & $b_{33}=0.0024263115650638677$ \\
\hline$a_{31}=-0.127049212625417$ & $a_{32}=-0.019024419473703036$ & $a_{33}=-0.01122301180646885$ \\
\hline$b_{41}=0.0752539843015807$ & $b_{42}=0.01604908927951054$ & $b_{43}=0.008383313974244766$ \\
\hline$a_{41}=-0.246331761062075$ & $a_{42}=-0.08287382942385146$ & $a_{43}=0$ \\
\hline$b_{51}=-0.0115113874206879$ & $b_{52}=-0.0052906035650492796$ & $b_{53}=0$ \\
\hline$a_{51}=0.357208872795928$ & $a_{52}=0.10037320724578758$ & $a_{53}=0$ \\
\hline$b_{61}=1 / 2-\left(b_{11}+\cdots+b_{51}\right)$ & $b_{62}=0.024232288421382347$ & $b_{63}=0$ \\
\hline$a_{61}=1-2\left(a_{11}+\cdots+a_{51}\right)$ & $a_{62}=0$ & $a_{63}=0.0014922318405735352$ \\
\hline
\end{tabular}

which results finally in

$$
\begin{aligned}
& \tilde{A}_{i}=\left(\frac{1}{2} a_{i 1}-\sqrt{3} a_{i 2}\right) h A_{1}+\left(\frac{1}{2} a_{i 1}+\sqrt{3} a_{i 2}\right) h A_{2} \\
& \tilde{B}_{i}=\left(\frac{1}{2} b_{i 1}-\sqrt{3} b_{i 2}\right) h B_{1}+\left(\frac{1}{2} b_{i 1}+\sqrt{3} b_{i 2}\right) h B_{2} .
\end{aligned}
$$

For future reference, note that $\mathrm{e}^{\tilde{A}_{i}}$ can be seen as the solution of the initial value problem $x^{\prime}=\hat{A}_{i} x, x(0)=I$ at $t=h$, where $\tilde{A}_{i}=h \hat{A}_{i}$. Of course, the same considerations apply to $\mathrm{e}^{\tilde{B}_{i}}$.

\subsection{Sixth-order methods}

In the general separable case, our standard splitting method is the symmetric composition $S_{10}$ with ten stages $(m=11)$ given in [6]. Therefore, the final scheme has the ABA form

$$
\mathrm{e}^{a_{11} \alpha_{1}+a_{12} \alpha_{2}+a_{13} \alpha_{3}} \mathrm{e}^{b_{11} \beta_{1}+b_{12} \beta_{2}+b_{13} \beta_{3}} \cdots \mathrm{e}^{b_{11} \beta_{1}-b_{12} \beta_{2}+b_{13} \beta_{3}} \mathrm{e}^{a_{11} \alpha_{1}-a_{12} \alpha_{2}+a_{13} \alpha_{3}} .
$$

We can distinguish two different situations.

- Only the conditions $\left[\alpha_{i}, \alpha_{j}\right]=\left[\beta_{i}, \beta_{j}\right]=0$ for $i \neq j$ are imposed. There are 22 order conditions for the 21 parameters $a_{i j}, b_{i j}, i>1$, whose structure admits in fact several solutions. With one of them we build the method $\mathrm{GS}_{10}-6$ of table 3 . 
- Suppose that, in addition, $A$ in (13) is constant, so that $\alpha_{i}=0$ for $i>1$. One possible solution in this case corresponds to the set of coefficients of $\mathrm{GS}_{10}-6$, but now with $a_{i 2}=a_{i 3}=0, i=1, \ldots, 6$. This solution stays very close to the solution which minimizes the sum of the absolute values of the coefficients, and shows very similar results in the numerical examples.

If, finally, $\left[\beta_{i}, \beta_{j}, \beta_{k}, \alpha_{l}\right]=0$ holds, we choose as the starting splitting scheme the 11stage $(m=12)$ symmetric RKN method $\mathrm{SRKN}_{11}^{b}$ of [6]. Now there are 20 order conditions and 23 parameters. A possible solution is shown in table 3 , and thus the method $\mathrm{MN}_{11}-6$

$$
\mathrm{e}^{b_{11} \beta_{1}+b_{12} \beta_{2}+b_{13} \beta_{3}} \mathrm{e}^{a_{11} \alpha_{1}+a_{12} \alpha_{2}+a_{13} \alpha_{3}} \ldots \mathrm{e}^{a_{11} \alpha_{1}-a_{12} \alpha_{2}+a_{13} \alpha_{3}} \mathrm{e}^{b_{11} \beta_{1}-b_{12} \beta_{2}+b_{13} \beta_{3}}
$$

is formed.

For the practical application of these methods, we use the linear relations (30) and (31) with a Gauss-Legendre quadrature rule, so that $s=k=3$. As a result we have

$$
\tilde{A}_{i}=h \hat{A}_{i} \equiv h \sum_{j=1}^{3} \rho_{i j} A_{j}, \quad \tilde{B}_{i}=h \hat{B}_{i} \equiv h \sum_{j=1}^{3} \sigma_{i j} B_{j}
$$

with $\rho_{i j}, \sigma_{i j}$ the elements of

$$
R^{(m, 3)} \equiv A^{(m, 3)} R^{(3)} Q_{G}^{(3,3)}, \quad S^{(m, 3)} \equiv B^{(m, 3)} R^{(3)} Q_{G}^{(3,3)},
$$

respectively. Expressions (39) are still valid (with different values of $\rho_{i j}, \sigma_{i j}$ ) if other quadrature rules are used instead.

\section{Methods for separable nonlinear systems}

As a matter of fact, the previous treatment can be easily generalized to the nonlinear equation (3) and the new splitting methods constructed in section 3 can be applied in this setting with only minor modifications.

If $\varphi_{t}$ denotes the exact flow of the differential equation $x^{\prime}=f(x, t)$, i.e., $x(t)=\varphi_{t}\left(x_{0}\right)$, then for each infinitely differentiable map $g: \mathbb{R}^{d} \rightarrow \mathbb{R}, g\left(\varphi_{t}(y)\right)$ admits the representation

$$
g\left(\varphi_{t}(y)\right)=\Phi_{t}[g](y),
$$

where $\Phi_{t}$ satisfies formally the time-dependent operator equation [5]:

$$
\frac{\mathrm{d}}{\mathrm{d} t} \Phi_{t}=\Phi_{t} L_{f(y, t)}, \quad y=x_{0} .
$$

Here $L_{f(y, t)}$ is the Lie operator associated with $f$, acting on differentiable functions. Just as for (13), we can now use the Magnus expansion to obtain the formal solution of (40):

$$
\Phi_{t}=\exp \left(L_{w\left(x_{0}, t\right)}\right)
$$

with $w=\sum_{i} w_{i}$. The first two terms read explicitly

$$
\begin{aligned}
& w_{1}\left(x_{0}, t\right)=\int_{t_{0}}^{t} f\left(x_{0}, s\right) \mathrm{d} s, \\
& w_{2}\left(x_{0}, t\right)=-\frac{1}{2} \int_{t_{0}}^{t} \mathrm{~d} s_{1} \int_{t_{0}}^{s_{1}} \mathrm{~d} s_{2}\left(f\left(x_{0}, s_{1}\right), f\left(x_{0}, s_{2}\right)\right),
\end{aligned}
$$

where the symbol $(f, g)$ stands for the Lie bracket [1 chapter 8],

$$
(f, g)_{i}=\sum_{j=1}^{d}\left(f_{j} \frac{\partial g_{i}}{\partial x_{j}}-g_{j} \frac{\partial f_{i}}{\partial x_{j}}\right) .
$$


The object $\exp \left(L_{w\left(x_{0}, t\right)}\right)$ is called the Lie transform associated with $w$. When $f$ does not depend explicitly on time, $\Phi_{t}$ is given simply by $\Phi_{t}=\exp \left(t L_{f}\right)$. It verifies the following important property: if $\varphi_{t}^{[j]}$ is the flow of a differential equation $y^{\prime}=f^{[j]}(y)$, then it is true that [10, p 84]

$g\left(\varphi_{t_{1}}^{[1]} \circ \varphi_{t_{2}}^{[2]} \circ \cdots \circ \varphi_{t_{n}}^{[n]}(y)\right)=\exp \left(t_{n} L_{f^{[n]}}\right) \cdots \exp \left(t_{2} L_{f^{[2]}}\right) \exp \left(t_{1} L_{f^{[1]}}\right)[g](y)$.

In other words, the Lie transforms appear in the reverse order as their corresponding maps. This also manifests itself in (40), where the linear operator $L_{f}$ is on the right side of $\Phi_{t}$.

We analyse now the separable problem (3). Let us define similarly to the linear case

$$
\alpha_{i}=\left.\frac{h^{i}}{(i-1) !} \frac{\partial^{i-1} f^{[A]}\left(x_{0}, t\right)}{\partial t^{i-1}}\right|_{t=t_{1 / 2}}, \quad \beta_{i}=\left.\frac{h^{i}}{(i-1) !} \frac{\partial^{i-1} f^{[B]}\left(x_{0}, t\right)}{\partial t^{i-1}}\right|_{t=t_{1 / 2}}
$$

and the corresponding integrals

$$
A^{(i)}=\frac{1}{h^{i}} \int_{-\frac{h}{2}}^{\frac{h}{2}} t^{i} f^{[A]}\left(x_{0}, t+t_{1 / 2}\right) \mathrm{d} t, \quad B^{(i)}=\frac{1}{h^{i}} \int_{-\frac{h}{2}}^{\frac{h}{2}} t^{i} f^{[B]}\left(x_{0}, t+t_{1 / 2}\right) \mathrm{d} t .
$$

Then, from the previous considerations, it is clear that the composition (26) corresponds now to

$$
\Psi_{m, h}^{[p]}=\prod_{i=m}^{1} \exp \left(L_{\tilde{B}_{i}}\right) \exp \left(L_{\tilde{A}_{i}}\right)=\exp \left(L_{w}\right)+\mathcal{O}\left(h^{p+1}\right),
$$

where $\tilde{A}_{i}\left(x_{0}\right), \tilde{B}_{i}\left(x_{0}\right)$ are given by (27) or (28) with exactly the same coefficients $a_{i j}, b_{i j}$. Observe that, due to property (44), the exponentials are disposed in the reverse order. When $\alpha_{i}, \beta_{i}$ are expressed in terms of $A^{(i)}, B^{(i)}$ and these integrals are approximated with quadrature rules we have

$$
\begin{aligned}
& \tilde{A}_{i}\left(x_{0}\right)=h \sum_{j=1}^{k}\left(R^{(m, k)}\right)_{i j} f^{[A]}\left(x_{0}, \tau_{j}\right) \\
& \tilde{B}_{i}\left(x_{0}\right)=h \sum_{j=1}^{k}\left(S^{(m, k)}\right)_{i j} f^{[B]}\left(x_{0}, \tau_{j}\right),
\end{aligned}
$$

where the matrices $R^{(m, k)}$ and $S^{(m, k)}$ are given by (31) and $\tau_{j}=t_{0}+c_{j} h$.

To evaluate the Lie transforms $\exp \left(L_{\tilde{A}_{i}\left(x_{0}\right)}\right)$ and $\exp \left(L_{\tilde{B}_{i}\left(x_{0}\right)}\right)$ is equivalent to compute the $h$-flow of the autonomous differential equations

$$
\begin{aligned}
x^{\prime} & =\frac{1}{h} \tilde{A}_{i}(x) \equiv \hat{A}_{i}(x) & x\left(t_{0}\right)=x_{0} \\
x^{\prime} & =\frac{1}{h} \tilde{B}_{i}(x) \equiv \hat{B}_{i}(x) & x\left(t_{0}\right)=x_{0}
\end{aligned}
$$

respectively. Let us denote these $h$-flows by $\varphi_{h}^{\left[\hat{A}_{i}\right]}, \varphi_{h}^{\left[\hat{B}_{i}\right]}$. Then, according to property (44), there exists a map

$$
\psi_{m, h}^{[p]}=\varphi_{h}^{\left[\hat{A}_{1}\right]} \circ \varphi_{h}^{\left[\hat{B}_{1}\right]} \circ \cdots \varphi_{h}^{\left[\hat{A}_{m}\right]} \circ \varphi_{h}^{\left[\hat{B}_{m}\right]},
$$

such that $g\left(\psi_{m, h}^{[p]}(y)\right)=\Psi_{m, h}^{[p]}[g](y)$. Note that the maps in (50) act in the same order as the exponentials of matrices for the linear problem in (26).

In summary, the application of the integrators proposed here involves the following steps.

(i) Select a particular splitting method among those built in section 3 for linear nonautonomous systems. This provides us with a set of coefficients $a_{i j}, b_{i j}$ and a specific distribution of exponentials in (26). 
(ii) For the separable nonlinear problem (3), compute the integrals $A^{(i)}(x), B^{(i)}(x)$ according to (46) or alternatively, choose a quadrature rule to approximate them to order $p$.

(iii) Compute $\tilde{A}_{i}(x), \tilde{B}_{i}(x), i=1, \ldots, m$, by (48).

(iv) Solve the autonomous nonlinear differential equations (49) and obtain $\varphi_{h}^{\left[\hat{A}_{i}\right]}, \varphi_{h}^{\left[\hat{B}_{i}\right]}, i=$ $1, \ldots, m$.

(v) To get the approximate solution at $t=t_{0}+h$, apply the composition (50) on the initial condition $x_{0}$. The sequence of maps in $\psi_{m, h}^{[p]}$ corresponds to the specific method selected in step (i).

Next we illustrate this procedure on a simple example.

Example. Let us consider equation (3) with $x=\left(x_{1}, x_{2}\right) \in \mathbb{R}^{2}$ and

$$
\begin{aligned}
& f^{[A]}(x, t)=\left(w_{1}(t) x_{1} x_{2}-w_{2}(t) x_{1}, 0\right) \\
& f^{[B]}(x, t)=\left(0, w_{3}(t) x_{2}-w_{4}(t) x_{1} x_{2}\right) .
\end{aligned}
$$

Our goal is to apply to this particular problem the sixth-order splitting method $\mathrm{GS}_{10}-6$, whose coefficients are given in table 3 (step (i)), with a Gauss-Legendre quadrature. In this case, then, $p=6, s=k=3$. For the step $t_{0} \mapsto t_{0}+h$, we evaluate first the time-dependent functions $w_{l}(t)$ at the quadrature nodes,

$$
w_{l j} \equiv w_{l}\left(\tau_{j}\right), \quad 1 \leqslant l \leqslant 4, \quad 1 \leqslant j \leqslant 3,
$$

which allows us to get $f^{[A]}\left(x, \tau_{j}\right), f^{[B]}\left(x, \tau_{j}\right)$ (step (ii)). Next we compute the vector fields $\tilde{A}_{i}, \tilde{B}_{i}$ according to

$$
\begin{array}{ll}
\tilde{A}_{i}(x)=h \sum_{j=1}^{3}\left(A^{(m, 3)} R^{(3)} Q_{G}^{(3,3)}\right)_{i j} f^{[A]}\left(x_{0}, \tau_{j}\right) & \\
\tilde{B}_{i}(x)=h \sum_{j=1}^{3}\left(B^{(m, 3)} R^{(3)} Q_{G}^{(3,3)}\right)_{i j} f^{[B]}\left(x_{0}, \tau_{j}\right), & 1 \leqslant i \leqslant m=11 .
\end{array}
$$

It is then clear that

$$
\tilde{A}_{i}(x)=\left(\tilde{w}_{1 i} x_{1} x_{2}-\tilde{w}_{2 i} x_{1}, 0\right), \quad \tilde{B}_{i}(x)=\left(0, \tilde{w}_{3 i} x_{2}-\tilde{w}_{4 i} x_{1} x_{2}\right)
$$

with

$$
\begin{array}{ll}
\left(\tilde{w}_{l 1}, \ldots, \tilde{w}_{l m}\right)^{T}=h A^{(m, 3)} R^{(3)} Q_{G}^{(3,3)}\left(w_{l 1}, w_{l 2}, w_{l 3}\right)^{T}, & l=1,2 \\
\left(\tilde{w}_{l 1}, \ldots, \tilde{w}_{l m}\right)^{T}=h B^{(m, 3)} R^{(3)} Q_{G}^{(3,3)}\left(w_{l 1}, w_{l 2}, w_{l 3}\right)^{T}, & l=3,4 .
\end{array}
$$

This completes step (iii). Now the solution of (49) is given by the maps (step (iv))

$$
\begin{aligned}
& \varphi_{h}^{\left[\hat{A}_{i}\right]}\left(x_{1}, x_{2}\right)=\left(x_{1} \exp \left(\left(\tilde{w}_{1 i} x_{2}-\tilde{w}_{2 i}\right) h\right), x_{2}\right) \\
& \varphi_{h}^{\left[\hat{B}_{i}\right]}\left(x_{1}, x_{2}\right)=\left(x_{1}, x_{2} \exp \left(\left(\tilde{w}_{3 i}-\tilde{w}_{4 i} x_{1}\right) h\right)\right), \quad 1 \leqslant i \leqslant m=11 .
\end{aligned}
$$

Finally, the application of scheme (50) for GS $\mathrm{GS}_{10}-6$ and the time step $t_{n}=t_{0}+n h \mapsto t_{n+1}$ with initial conditions $x_{1,0}=x_{1}\left(t_{n}\right), x_{2,0}=x_{2}\left(t_{n}\right)$ can be formulated by the algorithm (step $\left.(\mathrm{v})\right)$

$$
\begin{aligned}
& \text { do } i=1, m \\
& \qquad \begin{array}{l}
x_{2, i}=x_{2, i-1} \exp \left(h\left(\tilde{w}_{3, m+1-i}-\tilde{w}_{4, m+1-i} x_{1, i-1}\right)\right) \\
\quad x_{1, i}=x_{1, i-1} \exp \left(h\left(\tilde{w}_{1, m+1-i} x_{2, i}-\tilde{w}_{2, m+1-i}\right)\right) .
\end{array} \\
& \text { enddo }
\end{aligned}
$$

Note that in (50) the maps are computed from the right to the left and the algorithm can be written as an $m-1=10$ stage method because of the symmetry (33) and the FSAL property. 
For comparison, we include next the corresponding algorithm for the standard composition (2) and the splitting (4) based on the same coefficients $a_{i 1}, b_{i 1}$ of $\mathrm{GS}_{10}-6$. In this case two additional initial conditions are required, $x_{t 1,0}=t_{n}, x_{t 2,0}=t_{n}$ :

$$
\begin{aligned}
& \text { do } i=1, m \\
& \quad x_{2, i}=x_{2, i-1} \exp \left(h b_{m+1-i, 1}\left(w_{3}\left(x_{t 1, i-1}\right)-w_{4}\left(x_{t 1, i-1}\right) x_{1, i-1}\right)\right) \\
& \quad x_{t 2, i}=x_{t 2, i-1}+h b_{m+1-i, 1} \\
& \quad x_{1, i}=x_{1, i-1} \exp \left(h a_{m+1-i, 1}\left(w_{1}\left(x_{t 2, i}\right) x_{2, i}-w_{2}\left(x_{t 2, i}\right)\right)\right) \\
& \quad x_{t 1, i}=x_{t 1, i-1}+h a_{m+1-i, 1} . \\
& \text { enddo }
\end{aligned}
$$

Observe that in algorithm (53) each function $w_{l}(t)$ is evaluated $m$ times per step.

If the values of the functions $w_{k}(t)$ are known only at certain points (e.g. they are the solutions of other equations) it suffices for the first algorithm to choose an appropriate quadrature rule which considers the known points while for the second algorithm it is necessary to interpolate their values, with sufficient accuracy, at the points required by the algorithm.

\section{Numerical examples}

After discussing the practical implementation of the new splitting methods proposed in this work, it is time now to illustrate their performance and long-term behaviour on a pair of examples.

\subsection{The Lotka-Volterra model}

As is well known, the autonomous Lotka-Volterra model

$$
x_{1}^{\prime}=x_{1}\left(w_{1} x_{2}-w_{2}\right), \quad x_{2}^{\prime}=x_{2}\left(w_{3}-w_{4} x_{1}\right),
$$

with $w_{1}, w_{2}, w_{3}, w_{4}>0$ has periodic trajectories in the region $0<x_{1}, x_{2}$, around the point $\left(x_{1}, x_{2}\right)=\left(w_{3} / w_{4}, w_{2} / w_{1}\right)$, and also possesses the first integral $I\left(x_{1}, x_{2}\right)=$ $\ln \left(x_{1}^{w_{3}} x_{2}^{w_{2}}\right)-\left(w_{4} x_{1}+w_{1} x_{2}\right)$. The vector field $f\left(x_{1}, x_{2}\right)=\left(x_{1}\left(w_{1} x_{2}-w_{2}\right), x_{2}\left(w_{3}-w_{4} x_{1}\right)\right)$ is separable in two solvable parts, i.e. $f^{[A]}=\left(x_{1}\left(w_{1} x_{2}-w_{2}\right), 0\right)$ and $f^{[B]}=\left(0, x_{2}\left(w_{3}-w_{4} x_{1}\right)\right)$. If one considers the transformation $q=\ln \left(x_{1}\right), p=\ln \left(x_{2}\right)$, in the new variables the system is Hamiltonian with $H(q, p)=\left(w_{3} q-w_{4} e^{q}\right)+\left(w_{2} p-w_{1} e^{p}\right)$, which is also separable. In fact, both splittings are equivalent. In consequence, symplectic splitting methods for separable problems are suitable to numerically integrate this problem. Since neither the momenta part nor the coordinate part are quadratic, Nyström methods are not recommended [20]. The numerical solutions obtained by splitting symplectic integrators are also periodic trajectories of a perturbed Hamiltonian system, and their error grows more slowly than, in general, with non-symplectic methods.

Equations (54) are frequently used to describe the population of two species $x_{1}(t)$ (the number of predators) and $x_{2}(t)$ (the number of preys). External interactions (with other species, weather influence, etc) can be introduced in this model by taking time-dependent functions $w_{l}(t)$ in (54). Then we obtain precisely the example analysed in the previous section, with $f^{[A]}(x, t)$ and $f^{[B]}(x, t)$ given by (51). In particular, we may consider periodic functions

$$
\begin{array}{ll}
w_{1}(t)=1+\varepsilon \cos (2 t) & w_{3}(t)=1+\varepsilon \sin (t) \\
w_{2}(t)=2+\varepsilon \cos (t) & w_{4}(t)=1+\varepsilon \sin (2 t) .
\end{array}
$$

When $\varepsilon=0$ the autonomous problem (54) is recovered. For $|\varepsilon| \ll 1$, the trajectories stay in bounded regions of phase space. Since the perturbation is periodic, we may 

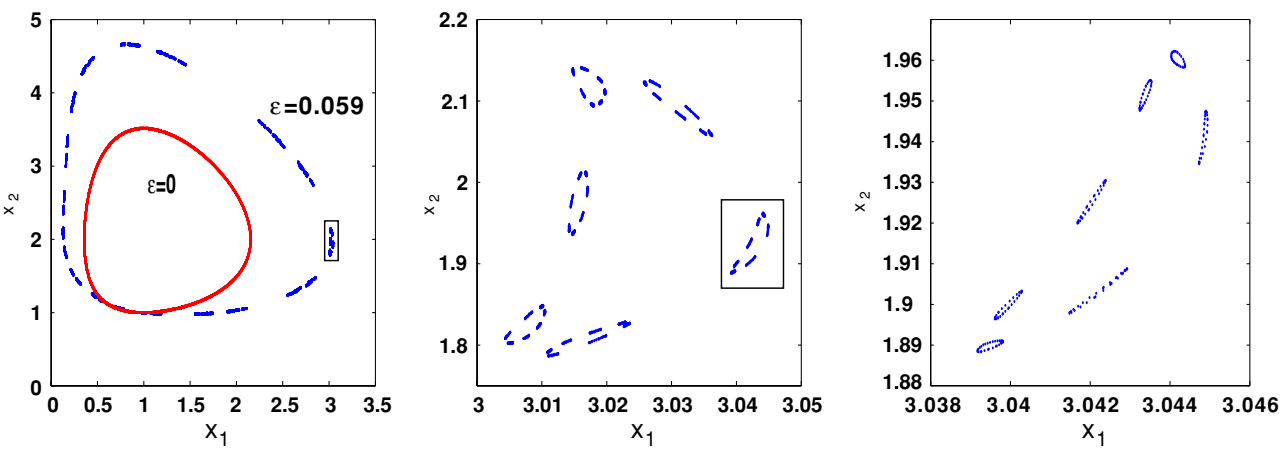

Figure 2. Poincaré map at $t=2 k \pi, k=0,1, \ldots, 20000$ for the Lotka-Volterra model and time-dependent functions given in (55).

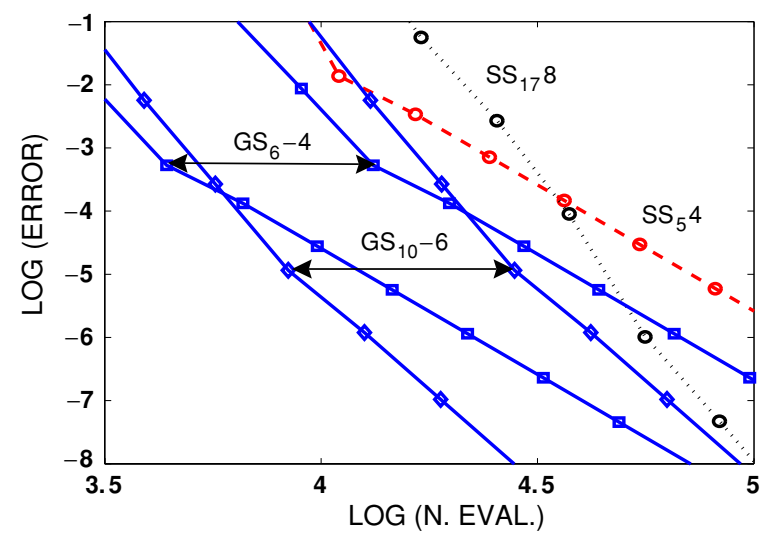

Figure 3. Efficiency curves for different splitting methods in the numerical integration of the Lotka-Volterra model with time-dependent functions (55) and $\varepsilon=0.059$. For the new methods $\mathrm{GS}_{6}-4$ and $\mathrm{GS}_{10}-6$ we consider as the cost both the number of stages (right curves) and the number of time-dependent function evaluations (left curves).

compute the Poincaré map at times $t=2 k \pi, k=0,1,2, \ldots$ We take as initial conditions $\left(x_{1,0}, x_{2,0}\right)=(1,1)$ and different values of $\varepsilon$ up to $\varepsilon \approx 0.06$, where the trajectories are unbounded. In figure 2 we show the Poincaré map for $\varepsilon=0$ (closed trajectory) and for $\varepsilon=0.059$ with 20000 points, i.e. at $t=2 k \pi, 0 \leqslant k \leqslant 20000$. The numerical computation is carried out with the sixth-order standard splitting method $S_{10}$ (applied to (4)) and the new scheme $\mathrm{GS}_{10}-6$. For this particular case the computational cost of $\mathrm{S}_{10}$ is nearly twice as costly as $\mathrm{GS}_{10}-6$, although this estimate obviously depends on the cost of the choice of the functions $w_{l}(t)$ of the model.

Next, for the case $\varepsilon=0.059$ we plot in figure 3 the work-accuracy curves obtained by different symplectic splitting methods, including the new integrators designed in this paper. To do that, we measure the average error in phase space at $t=2 k \pi, 0 \leqslant k \leqslant 100$, for different step sizes $h$.

In the numerical experiments, for the splitting (4) we take as the work per step the number of stages of the method, which, in general, corresponds to the number of evaluations of each $w_{l}(t)$. We show the results for $\mathrm{GS}_{6}-4$ and $\mathrm{GS}_{10}-6$ considering as the work per step the two extreme cases: (i) the cost is given by the number of stages (the solutions are very similar to 


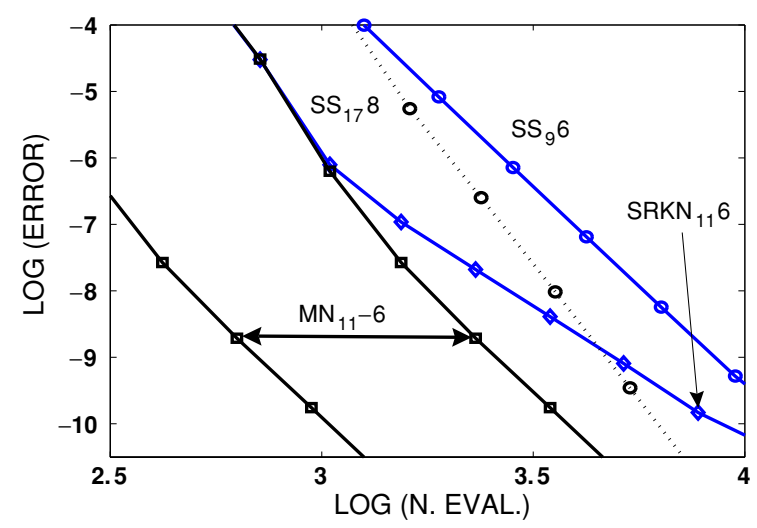

Figure 4. Same as figure 1(b), but including the results for the new sixth-order method for Nyström methods, $\mathrm{MN}_{11}-6$.

those obtained with the splitting (4) and taking $a_{i}=a_{i, 1}, b_{i}=b_{i, 1}$, which have not been plotted for clarity), and (ii) the cost is given by the number of time-dependent function evaluations (lines with squares and diamonds, respectively). Depending on the problem (the cost of the time-dependent functions and the cost of the map) the actual work-accuracy curves should stay between them. The results are compared with the scheme $\mathrm{SS}_{17} 8$ and the symmetricsymmetric five-stage fourth-order, $\mathrm{SS}_{5} 4$ due to Suzuki [22] (the curve obtained by scheme $\mathrm{SS}_{9} 6$ stay between them).

\subsection{The Duffing oscillator}

As our second example, we consider again the one-dimensional Duffing oscillator (6). We repeat the experiment shown in figure $1(b)$, but now including the new method results $\mathrm{MN}_{10}-6$, whose coefficients are collected in table 3 .

The corresponding results are shown in figure 4. As before, for the new method we take as its computational cost the two extreme cases previously enumerated. Observe that even in the least favourable situation the asymptotic behaviour of the scheme $\mathrm{MN}_{10}-6$ corresponds to a sixth-order method, as it should be. For this particular problem the fourth-order method $\mathrm{MN}_{6}-4$ exhibits a lower performance than $\mathrm{MN}_{10}-6$ for the whole range of accuracy shown in the figure, and so the corresponding curves have been omitted.

\section{Conclusions and outlook}

In this work, we have shown how to adapt splitting methods for autonomous separable systems to the more general case of explicitly time-dependent vector fields. The resulting new integration schemes are more efficient than standard methods applied to the enlarged system (4) on the numerical examples considered.

As a matter of fact, these methods can also be used on more relevant problems. Just as an example, note that the non-autonomous Duffing oscillator (6) can be written as

$$
q^{\prime \prime}=-\epsilon q^{\prime}+q-q^{3}+\delta \cos (\omega t)
$$

Thus one expects that the relative efficiency of the different integration methods analysed here should not change when applied to the more general problem

$$
q^{\prime \prime}=A(t) q^{\prime}+f(q, t) \quad q_{0}=q\left(t_{0}\right) \in \mathbb{R}^{l}
$$


with $A \in \mathbb{R}^{l \times l}$. This system describes, in particular, a system of coupled Duffing oscillators [19]. It is separable as

$$
\frac{\mathrm{d}}{\mathrm{d} t}\left\{\begin{array}{l}
q \\
v
\end{array}\right\}=\left\{\begin{array}{c}
v \\
A(t) v
\end{array}\right\}+\left\{\begin{array}{c}
0 \\
f(q, t)
\end{array}\right\} .
$$

Equivalently, one can also write

$$
q^{\prime}=M(t) p \quad p^{\prime}=-M(t)^{-1} f(q, t)
$$

with $p=M^{-1} q^{\prime}$ and $M(t)$ being the solution of the linear equation $M^{\prime}=A(t) M$. If $f(q, t)=-\nabla_{q} V(q, t)$ this second system of equations is nearly Hamiltonian. Both systems are separable in exactly solvable parts for the autonomous case (e.g. if time is frozen), and RKN methods can be used for their numerical integration.

It is worth mentioning that on the numerical examples shown in this work, the cost of the time-dependent functions is significant. However, there are some other important problems where this is not the case, as occurs, for example, in the numerical integration of the Schrödinger equation using Fourier methods. This problem is separable and the cost of the splitting methods is dominated by the number of stages because this number coincides with the number of Fourier transforms required by the method. In [8], splitting methods tailored for the autonomous problem of order $p=m$ with $m=4,6,8,10,12$ (the number of stages per step) are given. Their performance on the autonomous Schrödinger equation is superior to other splitting methods of similar order. However, if these methods are used for the non-autonomous problem (e.g. with a time-dependent potential) the order of all these methods reduces to $p=3$ making them useless. It is then noticeable to mention that the Magnus technique proposed in this work can also be used to recover the order these methods have for the autonomous problem. The Lie algebra has additional simplifications but the schemes in [8] are not symmetric so, in practice, we expect it is possible to build methods up to order 6 or 8 but, as mentioned, we can use the coefficients $a_{i, 1}, b_{i, 1}$ of a higher order method.

Another interesting problem corresponds to the near-integrable system, $x^{\prime}=f^{[A]}(x, t)+$ $\varepsilon f^{[B]}(x, t)$ with $\|\varepsilon\| \ll 1$. For the autonomous case, a number of highly efficient splitting methods can be found in [16]. If we consider the enlarged system (4) then both parts of the vector field are $\mathcal{O}(1)$ and the performance of these methods can be strongly reduced. The same procedure shown in this paper can also be used to build methods for this problem but taking into account a bi-graded Lie algebra in terms of powers of $h$ and $\varepsilon$. Then, these constitute interesting problems to be analysed in the future.

\section{Acknowledgments}

This work has been partially supported by Ministerio de Educación y Ciencia (Spain) under project MTM2004-00535 (co-financed by the ERDF of the European Union) and Generalitat Valenciana (GV2003-002). The work of SB has also been supported by a contract in the Programme Ramón y Cajal 2001.

\section{References}

[1] Arnold V I 1989 Mathematical Methods of Classical Mechanics 2nd edn (New York: Springer)

[2] Blanes S, Casas F and Ros J 2000 Improved high order integrators based on Magnus expansion BIT 40 434-50

[3] Blanes S, Casas F and Ros J 2002 High order optimized geometric integrators for linear differential equations BIT 42 262-84

[4] Blanes S and Moan P C 2000 Splitting methods for the time-dependent Schrödinger equation Phys. Lett. A $26535-42$ 
[5] Blanes S and Moan P C 2001 Splitting methods for non-autonomous Hamiltonian equations J. Comput. Phys. 170 205-30

[6] Blanes S and Moan P C 2002 Practical symplectic partitioned Runge-Kutta and Runge-Kutta-Nyström methods J. Comput. Appl. Math. 142 313-30

[7] Blanes S and Moan P C Fourth- and sixth-order commutator-free Magnus integrators for linear and non-linear dynamical systems Appl. Num. Math. (at press)

[8] Gray S and Manolopoulos D E 1996 Symplectic integrators tailored to the time-dependent Schrödinger equation J. Chem. Phys. 104 7099-112

[9] Guckenheimer J and Holmes P 1983 Nonlinear Oscillators, Dynamical Systems, and Bifurcations of Vector Fields (New York: Springer)

[10] Hairer E, Lubich C and Wanner G 2002 Geometric Numerical Integration. Structure-Preserving Algorithms for Ordinary Differential Equations (Berlin: Springer)

[11] Iserles A, Munthe-Kaas H Z, Nørsett S P and Zanna A 2000 Lie group methods Acta Numer. 9 215-365

[12] Iserles A and Nørsett S P 1999 On the solution of linear differential equations in Lie groups Phil. Trans. R. Soc. A 357 983-1019

[13] Leimkuhler B and Reich S 2004 Simulating Hamiltonian Dynamics (Cambridge: Cambridge University Press)

[14] Magnus W 1954 On the exponential solution of differential equations for a linear operator Commun. Pure Appl. Math. 7 649-73

[15] McLachlan R I 1995 On the numerical integration of ordinary differential equations by symmetric composition methods SIAM J. Sci. Comput. 16 151-68

[16] McLachlan R I 1995 Composition methods in the presence of small parameters BIT $35258-68$

[17] McLachlan R I and Quispel R 2002 Splitting methods Acta Numer. 11 341-434

[18] Munthe-Kaas H and Owren B 1999 Computations in a free Lie algebra Phil. Trans. R. Soc. A 357 957-81

[19] Paul Raj S, Rajasekar S and Murali K 1999 Coexisting chaotic attractors, their basin of attraction and synchronization of chaos in two coupled Duffing oscillators Phys. Lett. A $264283-8$

[20] Sanz-Serna J M and Calvo M P 1994 Numerical Hamiltonian Problems (London: Chapman \& Hall)

[21] Sanz-Serna J M and Portillo A 1966 Classical numerical integrators for wave-packet dynamics J. Chem. Phys. 104 2349-55

[22] Suzuki M 1990 Fractal decomposition of exponential operators with applications to many-body theories and Monte C arlo simulations Phys. Lett. A 146 319-23

[23] Varadarajan V S 1984 Lie Groups, Lie Algebras, and Their Representations (New York: Springer)

[24] Yoshida H 1990 Construction of higher order symplectic integrators Phys. Lett. A 150 262-8 